\title{
Proposta de um Descritor Híbrido para Aprimoramento da Identificação Automática de Melanoma
}

\author{
Nayara Moura, Rodrigo Veras, Kelson Aires, Luís Santos, Vinicius Machado \\ Departamento de Computação - Universidade Federal do Piauí (UFPI) \\ Campus Ministro Petrônio Portella - Teresina - PI - Brasil \\ naayaraholanda@gmail.com, rveraseufpi.edu.br
}

\begin{abstract}
Melanoma is skin cancer with a high degree of mortality. An estimated for 2017 that 9,730 people will die of melanoma in the USA. However, early detection of the disease reduces the death rate dramatically. Thus, this work aims to propose a hybrid descriptor for extraction of characteristics in the detection of melanoma. Several methods have been proposed, but most use features of a single descriptor. Thus, a combination of the main descriptors was performed and a selection of 10\% of the most relevant attributes with the Gain Ratio Information. The results obtained prove that the method is efficient, reaching an accuracy of $95.5 \%$ ( $\mathrm{PH}^{2}$ database) and $84.9 \%$ (DermIS database) with the MultiLayer Perceptron.
\end{abstract}

Resumo. O melanoma é o câncer de pele com alto grau de mortalidade. Estima-se para 2017 que 9.730 pessoas morrerão de melanoma nos EUA. Contudo, a detecção precoce da doença reduz a taxa de mortalidade drasticamente. Desse modo, esse trabalho tem como objetivo propor um descritor híbrido para extração de características na detecção do melanoma. Diversos métodos foram propostos, porém, em sua maioria utilizam características de um único descritor. Assim, foi realizada a combinação dos principais descritores e uma seleção de $10 \%$ dos atributos mais relevantes com o Gain Ratio Information. Os resultados obtidos provam que o método é eficiente, atingindo uma acurácia de 95,5\% (na base $\mathrm{PH}^{2}$ ) e 84,9\% (na base DermIS) com o MultiLayer Perceptron.

\section{Introdução}

O melanoma está entre os mais perigosos tipos de câncer devido à sua alta taxa de mortalidade. A estimativa de novos casos para o ano de 2016, no Brasil, foi de 5.670, sendo 3.000 homens e 2.670 mulheres [INCA 2016]. Apenas nos Estados Unidos, a taxa de mortalidade causada pelo melanoma é de $75 \%$ quando comparada à causada por cânceres de pele não-melanoma. Entretanto, nesse tipo de câncer, se detectado nos estágios iniciais, o prognóstico pode ser considerado bom [Society 2015].

A análise de imagens dermatoscópicas pelos especialistas é baseada em uma inspeção visual das manchas ou feridas da pele, verificando se aparentam com feridas que não cicatrizam, se possuem crescimento assimétrico, bordas não delimitadas, entre outros aspectos [Lacy e Alwan 2013]. Um exemplo é a regra ABCD (Assimetria, Borda, Cor, Diâmetro), que também é utilizada por diversos sistemas CAD (Computer Aided Diagnosis - Diagnóstico Assistido por Computador). 
A detecção precoce do melanoma ainda é um desafio e requer bastante atenção. $\mathrm{Na}$ literatura, muitos trabalhos nessa área utilizam um único descritor para a análise de imagens dermatoscópicas. Entretanto, essa pode não ser a estratégia ideal. Assim, o método proposto nesse trabalho é um descritor híbrido a partir da união de diferentes descritores como forma de alcançar uma melhor estratégia para a etapa de extração de características de sistemas CAD no problema estudado.

Foi realizada uma investigação dos principais descritores na identificação do melanoma e uma fusão dos mesmos com o propósito de combinar as características mais importantes. O objetivo é o desenvolvimento de um descritor que caracterize corretamente as lesões de pele como melanoma ou não-melanoma, com a finalidade de ser utilizado em um sistema CAD, auxiliando o dermatologista no diagnóstico.

O restante do artigo está organizado da seguinte forma: Seção 2 apresenta os trabalhos relacionados; Seção 3 descreve as bases de imagens utilizadas para os testes, os descritores e classificadores avaliados e o método proposto; Seção 4 apresenta os resultados e discussões; e por fim, Seção 5, a conclusão e trabalhos futuros.

\section{Trabalhos Relacionados}

Nos últimos anos, diversos trabalhos foram realizados em análise de imagens dermatoscópicas para detecção do melanoma, incluindo segmentação, extração de características e classificação. A extração de características pode ser vista como um passo fundamental para desenvolver um bom sistema CAD.

No trabalho de Chang et al. [Chang et al. 2013], um sistema CAD é proposto com o objetivo de diagnosticar lesões melanocíticas e não melanocíticas baseado em imagens fotográficas digitais convencionais. As características das imagens são extraídas através de descritores de cor, forma e textura em um total de 769 imagens de lesões de pele. Os algoritmos de textura utilizados foram a GLRLM (Gray Level Run Length Matrix) e de Tamura. Os resultados mostram que o método proposto alcançou uma acurácia de 90,64\% e uma sensibilidade de $85,63 \%$.

Em Codella et al. [Codella et al. 2015], Deep Learning e Sparce Coding são utilizados na extração de características de imagens de lesão de pele. Para a classificação, o algoritmo utilizado é o Support Vector Machine (SVM). Os experimentos foram realizados com uma quantidade de 2624 imagens (334 melanomas, 144 nevos atípicos e 2146 lesões benignas) e divididos em duas tarefas: diferenciar o melanoma das lesões não-melanoma e diferenciá-lo somente dos nevos atípicos. A primeira tarefa atingiu uma acurácia de 93,1\% e, a segunda, uma acurácia de 73,9\%.

No trabalho proposto por Bhati e Singhal [Bhati e Singhal 2015], a classificação de lesão de pele como benigna ou maligna para detecção do melanoma é feita a partir de um sistema baseado em TDS (Total Dermoscopic Score) e utiliza as características ABCD como descritor da lesão. A base de imagem é formada por 30 imagens (16 malignas e 14 benignas) de lesão de pele obtidas pelo Cancer Research Center, Binaker. Os resultados foram analisados de acordo com a sensibilidade e especificidade, alcançando 92,30\% e $84,61 \%$, respectivamente. Os pontos negativos desse trabalho está nas poucas imagens que foram utilizadas para validar o método proposto.

Já Sánchez-Monedero et al. [Sánchez-Monedero et al. 2016], propuseram um sis- 
tema que combina a regra ABCD, a GLCM (Grey-Level Co-occurrence Matrix), o LBP (Local Binary Pattern) e MRF (Markov Random Field) para a extração de características das lesões de pele. Nesse trabalho, a base de dados é composta por 556 imagens. Vários métodos de classificação foram utilizados para os experimentos. O melhor resultado encontrado foi uma acurácia de 68,51\% com o método LIPU (Logistic regression using Initial variables and Product Units).

Oliveira et al. [Oliveira et al. 2016] apresentam uma nova abordagem para extração de características de lesão de pele. A regra ABCD e o método Box-Counting são utilizados como descritores das imagens. Entretanto, o Diâmetro da regra ABCD não foi aplicado devido à sua grande dependência da resolução da imagem. As bases de imagens utilizadas possui um total de 408 imagens. Os experimentos foram divididos em três: classificação entre imagens de nevos e ceratose seborreica, entre nevos e melanoma, e entre ceratose seborreica e melanoma. Neste último, o resultado da classificação não foi tão expressivo com uma acurácia de 74,33\%, utilizando o SVM. O melhor resultado obtido foi uma acurácia de 79,01\%, porém, para a diferenciação entre nevos e ceratose seborreica, o que não torna tão eficiente o método.

\section{Materiais e Métodos}

\subsection{Bases de Imagens}

As bases de imagens utilizadas para os testes dos algoritmos constituem um total de 406 imagens dermatoscópicas. Essas bases foram escolhidas devido serem utilizadas em diversos trabalhos e principalmente diagnosticadas por um especialista, apresentando a máscara binária de cada lesão.

A base $\mathrm{PH}^{2}$ [Mendonça et al. 2013] é formada por 200 imagens, sendo elas, 80 nevos comuns, 80 nevos atípicos e 40 melanomas. A base DermIS (Dermatology Information System) [Diepgen e Yihune 2012] possui um total de 206 imagens dermatoscópicas, divididas entre melanoma e não melanoma, 119 e 87, respectivamente. A Figura 1 apresenta exemplos de imagens de lesão de pele dessas duas bases utilizadas nesse trabalho.

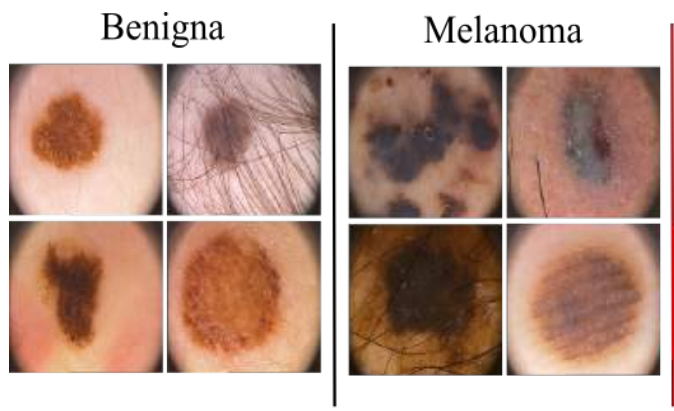

(a)

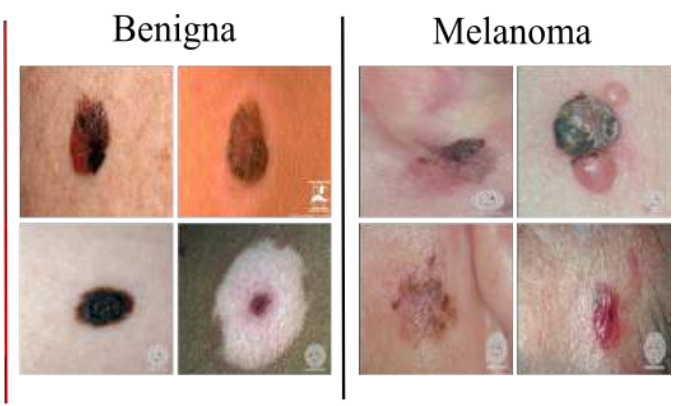

(b)

Figura 1. Exemplos de imagens das bases $\mathrm{PH}^{2}$ (a) e DermIS (b), de acordo com a categoria.

\subsection{Descritores}

Atributos são propriedades que podem ser mensuradas a partir de uma imagem, como por exemplo forma, cor e textura. Esses atributos, agrupados em um vetor de características, 
são denominados de descritor de imagem. Os descritores utilizados para extrair características de imagens de lesão de pele e formar o descritor híbrido proposto são detalhados a seguir:

Regra ABCD: Essa regra é dividida em quatro etapas para descrever a imagem e retorna 5 atributos: Assimetria (1), Irregularidade da Borda (2), Cor (1) e Diâmetro (1). A primeira característica calculada é o Diâmetro. Inicialmente, com a máscara binária, $o$ contorno da lesão é obtido pelo algoritmo de Sobel [Sobel 1970], Em seguida, é calculada a maior distância entre dois pontos $\left(p_{1}=\left(x_{1}, y_{1}\right)\right.$ e $\left.p_{2}=\left(x_{2}, y_{2}\right)\right)$ (diagonal maior) pertencentes à borda da lesão através do uso da equação da distância euclidiana (Equação 1). O resultado, em pixels, é utilizado para a classificação.

$$
D_{\left(p_{1}, p_{2}\right)}=\sqrt{\left(x_{1}-x_{2}\right)^{2}+\left(y_{1}-y_{2}\right)^{2}} .
$$

A Assimetria é encontrada a partir do Diâmetro, onde a maior distância, em pixels, entre a reta (diagonal maior) e um ponto pertencente à borda é obtida pela Equação 2. Onde $a, b, c$ são os coeficientes da reta e $x_{0}, y_{0}$ os pontos da coordenada da imagem.

$$
d=\frac{\left|a x_{0}+b y_{0}+c\right|}{\sqrt{a^{2}+b^{2}}}
$$

A Irregularidade da Borda é calculada a partir de duas técnicas. Na primeira, pesos são atribuídos para os quatro vizinhos da direita e da esquerda de todos os pontos da borda da lesão. O vizinho que estiver acima do ponto analisado em relação à coordenada $y$, recebe peso -1 , caso contrário, recebe peso 1 . Se esta soma for maior ou igual a 2 ou menor ou igual a -2 , e positiva, a inflexão é considerada um pico, mas se negativa, é considerada um vale. $\mathrm{O}$ objetivo é descobrir a quantidade total de picos e vales menores na lesão [Araujo et al. 2012].

Já na segunda técnica, encontra-se os picos e vales maiores da lesão. O produto vetorial é empregado para todos os pontos da borda da lesão, considerando os seus vizinhos da direita e esquerda $(\mathrm{p}-15 ; \mathrm{p} ; \mathrm{p}+15)$, sendo $p$ o ponto, ou seja, variação de 15 pixels à esquerda e 15 pixels à direita do ponto $p$. Assim, caso o resultado do produto vetorial seja maior que zero, $p_{1}=\left(x_{1}, y_{1}\right), p_{2}=\left(x_{2}, y_{2}\right)$ e $p_{3}=\left(x_{3}, y_{3}\right)$ constituem um pico e, se menor que zero, constituem um vale [Araujo et al. 2012].

Na característica Cor, a quantidade de cores encontradas na lesão é utilizada na classificação. Inicialmente, é realizada uma divisão da imagem em dez intervalos (cores pertencentes ao mesmo foram consideradas semelhantes). Para cada intervalo, é feita a contagem do número de pixels. Entretanto, as regiões muito pequenas (menos de 100 pixels) foram desconsideradas [Araujo et al. 2012].

Grey-Level Co-occurrence Matrix (GLCM): A GLCM, ou Matrizes de Coocorrências de Níveis de Cinza [Haralick et al. 1973] é um descritor de textura que analisa as co-ocorrências entre pares de pixels e armazena suas intensidades relativas em uma matriz quadrada. As probabilidades de co-ocorrências são calculadas entre dois níveis de cinza $i$ e $j$, utilizando uma orientação $\theta$ (theta) $\left(0^{\circ}, 45^{\circ}, 90^{\circ}\right.$ ou $\left.135^{\circ}\right)$ e uma distância conhecida como pixel pair spacing. 
Várias características são definidas para a GLCM, porém foram utilizadas as mais importantes: contraste, correlação, energia, homogeneidade e entropia. Uma vez que, a utilização de algumas dessas características pode gerar um melhor resultado do que a utilização de todas [Silva Júnior 2007]. Assim, são retornados 17 atributos.

Gray Level Run Length Matrix (GLRLM): A GLRLM, ou Método de Comprimento de Primitiva a Nível de Cinza, descritor de textura, utiliza matrizes auxiliares e verifica as repetições dos pixels (primitivas). Uma matriz GLRLM é criada para cada corte e possui o número de primitivas dos níveis de cinza na direção $\theta\left(0^{\circ}, 45^{\circ}, 90^{\circ}\right.$ ou $135^{\circ}$ ) e a uma distância $d$. Assim, esse descritor retorna 44 características. A soma de todas as matrizes GLRLM de cada corte é feita, e a partir do número de repetições encontrados, a matriz é dividida e normalizada.

Segundo Galloway [Galloway 1975], a partir da matriz do GLRLM cinco medidas estatísticas podem ser extraídas. São elas, a ênfase em primitivas curtas (Short-Run Emphasis), a ênfase em primitivas longas (Long-Run Emphasis), a uniformidade do nível de cinza (Gray Level distribution), a uniformidade do comprimento da primitiva (Run Length distribution) e a percentagem de primitivas (Run Percentage). Entretanto, mais duas medidas estatísticas foram determinadas por Chu et al. [Chu et al. 1990], a ênfase em primitivas de baixo nível de cinza (Low Gray Level Run Emphasis) e a ênfase em primitivas de alto nível de cinza (High Gray Level Run Emphasis).

Outras quatro funções de extração de características seguindo a ideia das medidas estatísticas foram descritas por Dasarathyand e Holder [Dasarathyand e Holder 1991]. São elas: ênfase em primitivas curtas de baixo nível de cinza (Short Run Low Gray Level Emphasis), ênfase em primitivas curtas de alto nível de cinza (Short Run High Gray Level Emphasis), ênfase em primitivas longas de baixo nível de cinza (Long Run Low Gray Level Emphasis) e ênfase em primitivas longas de alto nível de cinza (Long Run High Gray Level Emphasis).

Histograms of Oriented Gradients (HOG): O Histograma de Gradientes Orientados [Dalal e Triggs 2005], calcula o histograma da orientação dos gradientes na imagem. Ele converte a imagem para escala de cinzas e a normaliza de acordo com a iluminação. A computação dos gradientes e uma divisão da imagem em pequenas regiões espaciais chamadas células são realizadas.

Para cada célula é calculado um histograma local 1-D das orientações sobre os pixels da célula. Após os histogramas serem computados, eles são normalizados através de um acúmulo de histogramas locais em regiões espaciais um pouco maiores chamadas de blocos. Os histogramas acumulados são então utilizados para normalizar todas as células naquele bloco. Após a normalização, uma janela de detecção é coletada sobre os histogramas gerados, que consiste na saída do descritor HOG com 81 atributos.

Local Binary Pattern (LBP): No descritor de textura LBP, ou Padrão Binário Local [Ojala et al. 1996], é realizada uma rotulação dos pixels de uma imagem em níveis de cinza por limiarização. Cada pixel é comparado com o central da janela de vizinhança 3 x 3 que constitui o valor do limiar. Quando todas as janelas forem computadas, o vetor de atributos (com 256 atributos) é montado com a junção dos valores obtidos. Assim, o histograma da célula é calculado, ou seja, as quantidades de " 0 "e " 1 ".

Tamura: Proposto por Tamura [Tamura et al. 1978], esse descritor de textura cal- 
cula sob a imagem as seguintes três características: coarseness, contraste e direcionalidade. Essas características estão relacionadas à percepção visual humana.

Box-Counting: O método Box-Counting também é um descritor de textura, que calcula as dimensões fractais [Al-Akaidi 2004] sob uma imagem, retornando 11 atributos. A dimensão fractal é um processo que divide a imagem em vários quadrantes para quantificar o nível de irregularidade ou auto-similaridade fractal da mesma.

Convolutional Neural Network (CNN): A CNN ou Rede Neural Convolucional utilizada nesse trabalho, possui a técnica de transferência de aprendizado proposta por Shin et al. [Shin et al. 2016]. Uma das formas de se realizar esta transferência, e a empregada nesse trabalho, é utilizando a penúltima camada da rede como matriz de características, contendo 4096 atributos por instância. As arquiteturas utilizadas foram: AlexNet, CaffeNet e VGGNet.

\subsection{Classificadores}

Um classificador tem por objetivo dividir o espaço de características em regiões de decisão. A entrada dos classificadores é o vetor de atributos resultante dos descritores, e a saída é a classe que a região pertence. Os classificadores que foram utilizados nesse trabalho são apresentados a seguir:

MultiLayer Perceptron (MLP): O MLP , ou Perceptron de Múltiplas Camadas, é uma rede neural artificial utilizada para resolver problemas mais complexos. Ele possui uma rede de perceptrons dispostos em camada. Seu método mais popular para aprendizado é chamado de retropropagação (backpropagation). Esse tipo de aprendizado consiste em dois passos através das diferentes camadas da rede: propagação e retropropagação. No primeiro, um padrão de atividades é aplicado aos nós sensoriais e a resposta real da rede é produzida. No segundo, os pesos sinápticos são ajustados de acordo com um sinal de erro para ser propagado na direção contrária das conexões sinápticas [Haykin 2009].

Support Vector Machine (SVM): Proposto por Cortes e Vapnik [Cortes e Vapnik 1995], a Support Vector Machine, ou Máquina de Vetor de Suporte, é uma técnica de aprendizagem supervisionada, que contém as fases de treinamento, atributos e saídas desejadas, e teste, para encontrar uma saída a partir de vetor de entrada. Em geral, é feito um mapeamento do espaço de entrada para um espaço de dimensionalidade maior e calculado, em seguida, um hiperplano de separação ótimo a fim de maximizar a distância de separação entre as classes.

Random Forest: O classificador Random Forest [Breiman 2001] é uma combinação de diversas árvores onde cada uma de suas árvores depende dos valores de um vetor independente aleatoriamente e com a mesma distribuição para todas as árvores da série de árvores de decisão. Por fim, quando um grande número de árvores são geradas, as classes com maior número de votos são eleitas.

Na geração dos resultados foi utilizado o método estatístico $k$-fold cross-validation $(k=10)$. E para avaliar o desempenho dos algoritmos foram utilizadas algumas métricas conhecidas na literatura: Precisão (P), Sensibilidade (S), índice Kappa (K) [Rosenfield e Fitzpatrick-Lins 1986] e Acurácia (A). 


\subsection{Método Proposto}

A Figura 2 apresenta o fluxograma do método proposto nesse trabalho. Inicialmente, todas as imagens foram convertidas para o sistema de cores HSV (Hue - Matiz, Saturation - Saturação, Value - Brilho). Em seguida, as características foram extraídas pelos descritores a partir da componente gerada pela soma das bandas S e V. Isso se deve ao fato que essa nova componente consegue diferenciar melhor os tipos de lesão de pele [Moura et al. 2015].

Uma seleção de atributos com o algoritmo Razão de Ganho de Informação (Gain Ratio Information) foi realizada no vetor de características obtido. Ele é um filtro de atributos supervisionado que pode ser usado para selecionar atributos. É muito flexível e permite combinar vários métodos de pesquisa e avaliação. $\mathrm{O}$ método utilizado foi o Ranker, que classifica os atributos por suas avaliações individuais. Depois dos atributos classificados, somente $10 \%$ deles foram utilizados. Visto que os descritores utilizados retornam uma grande quantidade de atributos (12.705 no total), ou seja, uma grande possibilidade de existir atributos que não sejam significativos ou que sejam duplicados, dificultando a classificação das lesões de pele [Kumar et al. 2016].

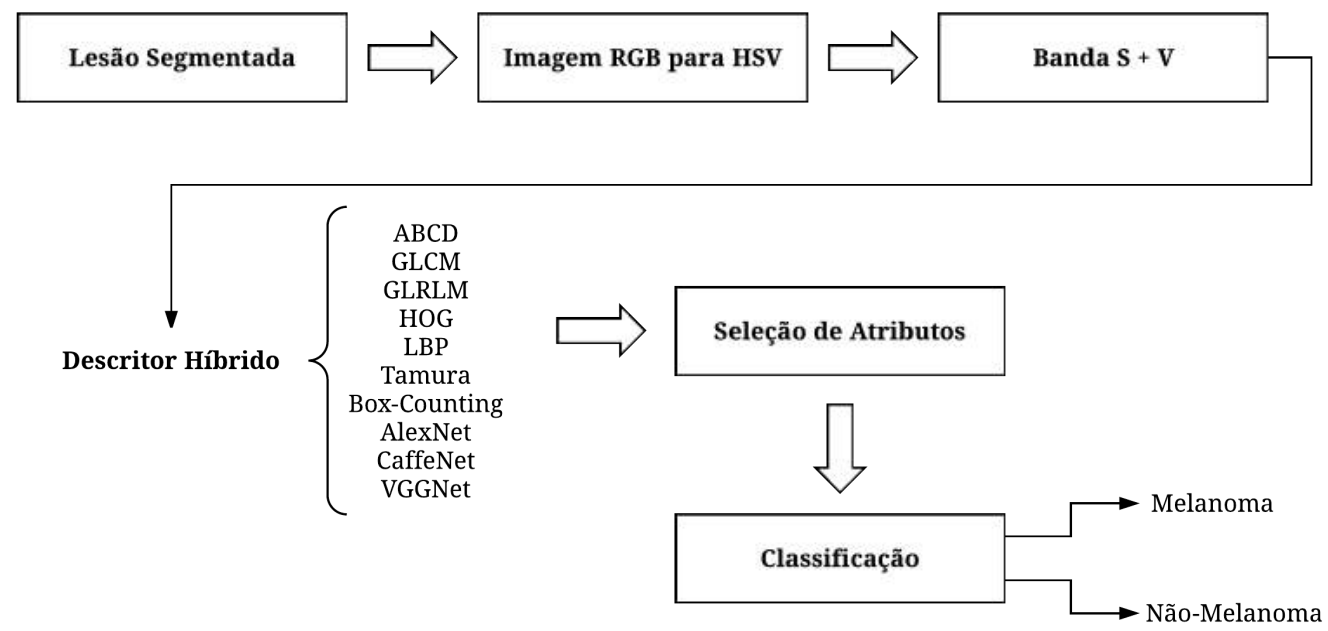

Figura 2. Fluxograma do método proposto para identificação de melanoma em imagens de lesão de pele.

\section{Resultados e Discussão}

As bases de imagens $\mathrm{PH}^{2}$ e DermIS utilizadas para os testes dos algoritmos constituem 200 e 206 imagens dermatoscópicas, respectivamente. Inicialmente, os testes foram feitos para cada um dos descritores apresentados separadamente, com o objetivo de realizar uma análise comparativa com o método proposto. Subsequentemente, os testes foram realizados no descritor híbrido proposto (união de todos os descritores). Para a classificação, os algoritmos SVM, MLP e Random Forest foram os escolhidos.

A Tabela 1 mostra os resultados alcançados na classificação nas imagens da base $\mathrm{PH}^{2}$ para cada um dos descritores. O CNN com a arquitetura VGG, utilizando o classificador SVM, obteve uma acurácia e sensibilidade de 92\%, com um índice Kappa de 0,724, considerado como um resultado "Muito Bom"(de acordo com o nível de exatidão de uma 
Tabela 1. Resultados da classificação das lesões de pele da base $\mathbf{P H}^{2}$.

\begin{tabular}{c|cccc|cccc|cccc}
\hline \multirow{2}{*}{ Método } & \multicolumn{4}{|c|}{ MLP } & \multicolumn{4}{c|}{ SVM } & \multicolumn{4}{c}{ Random Forest } \\
\cline { 2 - 14 } & $\mathrm{P}$ & $\mathrm{S}$ & $\mathrm{K}$ & $\mathrm{A}$ & $\mathrm{P}$ & $\mathrm{S}$ & $\mathrm{K}$ & $\mathrm{A}$ & $\mathrm{P}$ & $\mathrm{S}$ & $\mathrm{K}$ & $\mathrm{A}$ \\
\hline ABCD & 0,894 & 0,895 & 0,618 & 89,5 & 0,765 & 0,805 & 0,118 & 80,5 & 0,857 & 0,865 & 0,539 & 86,5 \\
\hline GLCM & 0,789 & 0,795 & 0,341 & 79,5 & 0,732 & 0,795 & 0,072 & 79,5 & 0,819 & 0,830 & 0,426 & 83 \\
\hline GLRLM & 0,885 & 0,890 & 0,636 & 89 & 0,640 & 0,800 & 0 & 80 & 0,885 & 0,890 & 0,636 & 89 \\
\hline HOG & 0,898 & 0,895 & 0,681 & 89,5 & 0,898 & 0,895 & 0,680 & 89,5 & 0,875 & 0,880 & 0,603 & 88 \\
\hline LBP & 0,863 & 0,870 & 0,522 & 87 & 0,640 & 0,800 & 0 & 80 & 0,845 & 0,855 & 0,449 & 85,5 \\
\hline Tamura & 0,891 & 0,895 & 0,634 & 89,5 & 0,885 & 0,890 & 0,628 & 89 & 0,820 & 0,815 & 0,438 & 81,5 \\
\hline Box-Counting & 0,876 & 0,860 & 0,6 & 86 & 0,876 & 0,860 & 0,6 & 86 & 0,876 & 0,876 & 0,876 & 86 \\
\hline CNN - AlexNet & 0,857 & 0,865 & 0,539 & 86,5 & 0,896 & 0,900 & 0,655 & 90 & 0,902 & 0,905 & 0,669 & 90,5 \\
\hline CNN - CaffeNet & 0,873 & 0,880 & 0,586 & 88 & 0,896 & 0,900 & 0,662 & 90 & 0,899 & 0,900 & 0,640 & 90 \\
\hline CNN - VGGNet & 0,873 & 0,880 & 0,586 & 88 & 0,919 & 0,920 & 0,724 & 92 & 0,907 & 0,910 & 0,689 & 91 \\
\hline Proposto & $\mathbf{0 , 9 5 4}$ & $\mathbf{0 , 9 5 5}$ & $\mathbf{0 , 8 5 5}$ & $\mathbf{9 5 , 5}$ & 0,640 & 0,800 & 0 & 80 & 0,945 & 0,945 & 0,816 & 94,5
\end{tabular}

classificação, estabelecido por Landis e Koch [Landis e Koch 1977]). Em contraste, o GLRLM, o LBP e o método proposto, também com o SVM, não conduziram a uma boa diferenciação entre as classes, um Kappa de 0 foi obtido, ou seja, o classificador errou todas imagens.

No entanto, o melhor resultado pode ser obtido com o método proposto, utilizando o classificador MLP, onde foi possível acertar 95,5\% das lesões de pele, atingindo também uma sensibilidade de 95,5\%, uma precisão de 95,4\% e um índice Kappa de 0,855, considerado "Excelente". Com o classificador Random Forest, o melhor resultado também foi alcançado com o método proposto, obtendo uma acurácia de 94,5\% e um índice Kappa de 0,816 .

A Tabela 2 apresenta os resultados obtidos a partir das imagens da base de dados DermIS. Para essa base, o melhor resultado de classificação também pode ser obtido com método proposto e o classificador MLP, atingindo uma acurácia de 84,9\% e um índice Kappa de 0,69, considerado "Muito Bom".

Com o descritor CNN, para todas as arquiteturas, um resultado com valor bem próximo foi obtido, utilizando o classificador SVM. Entretanto, ainda sim, o método proposto apresenta os melhores resultados quando utilizado com o classificador MLP. Ainda nessa base, o Random Forest com o descritor CNN e rede VGG atingiram uma acurácia de $83 \%$ e um Kappa de 0,645 .

Analisando os resultados obtidos para classificação de lesões de pele, é possível observar que o método proposto obteve o melhor valor de classificação em relação à todas as taxas com o classificador MLP. Isso se deve ao fato que a combinação de diversos algoritmos conseguiu diferenciar melhor as classes estudadas.

\section{Conclusão e Trabalhos Futuros}

Existem diversos métodos na literatura para classificação de lesões de pele. Entretanto, os resultados do método proposto se mostraram bastante satisfatórios em conjunto com o classificador MLP. O mesmo obteve uma acurácia de 95,5\% para a base $\mathrm{PH}^{2}$ e $84,9 \%$ para a base DermIS. Os resultados mostram que a combinação de vários algoritmos de extração de características descreve a imagem suficientemente bem para o problema em questão. Ou seja, isso implica dizer que o método proposto constitui um bom descritor para o classificador conseguir separar as duas classes. 
Tabela 2. Resultados da classificação das lesões de pele da base DermIS.

\begin{tabular}{c|cccc|cccc|cccc}
\hline \multirow{2}{*}{ Método } & \multicolumn{4}{|c|}{ MLP } & \multicolumn{4}{c|}{ SVM } & \multicolumn{4}{c}{ Random Forest } \\
\cline { 2 - 14 } & $\mathrm{P}$ & $\mathrm{S}$ & $\mathrm{K}$ & $\mathrm{A}$ & $\mathrm{P}$ & $\mathrm{S}$ & $\mathrm{K}$ & $\mathrm{A}$ & $\mathrm{P}$ & $\mathrm{S}$ & $\mathrm{K}$ & $\mathrm{A}$ \\
\hline ABCD & 0,525 & 0,553 & 0,021 & 55,3 & 0,448 & 0,519 & $-0,080$ & 51,9 & 0,476 & 0,476 & $-0,074$ & 47,5 \\
\hline GLCM & 0,665 & 0,660 & 0,257 & 66 & 0,718 & 0,675 & 0,271 & 67,4 & 0,595 & 0,602 & 0,166 & 60,1 \\
\hline GLRLM & 0,658 & 0,660 & 0,269 & 66 & 0,581 & 0,592 & 0,134 & 59,2 & 0,608 & 0,612 & 0,196 & 61 \\
\hline HOG & 0,686 & 0,689 & 0,345 & 68,9 & 0,334 & 0,578 & 0 & 57,7 & 0,630 & 0,636 & 0,233 & 63,5 \\
\hline LBP & 0,619 & 0,626 & 0,210 & 62,6 & 0,334 & 0,578 & 0 & 57,7 & 0,625 & 0,631 & 0,224 & 63,1 \\
\hline Tamura & 0,640 & 0,641 & 0,261 & 64 & 0,334 & 0,578 & 0 & 57,7 & 0,616 & 612 & 0,211 & 61,1 \\
\hline Box-Counting & 0,488 & 0,549 & $-0,02$ & 54,8 & 0,334 & 0,578 & 0 & 57,7 & 0,484 & 0,485 & $-0,057$ & 48,5 \\
\hline CNN - AlexNet & 0,811 & 0,811 & 0,612 & 81 & 0,850 & 0,850 & 0,688 & 84,9 & 0,835 & 0,835 & 0,657 & 83,4 \\
\hline CNN - CaffeNet & 0,836 & 0,835 & 0,662 & 83,4 & 0,849 & 0,850 & 0,689 & 84,9 & 0,805 & 0,806 & 0,598 & 80,5 \\
\hline CNN - VGGNet & 0,849 & 0,850 & 0,689 & 84,9 & 0,839 & 0,840 & 0,670 & 83,9 & 0,831 & 0,830 & 0,645 & 83 \\
\hline Proposto & $\mathbf{0 , 8 5 1}$ & $\mathbf{0 , 8 5 0}$ & $\mathbf{0 , 6 9}$ & $\mathbf{8 4 , 9}$ & 0,334 & 0,578 & 0 & 57,7 & 0,815 & 0,816 & 0,619 & 81,5
\end{tabular}

Os resultados apresentados são promissores. Entretanto, podem ser melhorados. Como trabalhos futuros, um passo importante para aumentar as taxas de acerto obtidas reside na adição de mais descritores de textura no descritor híbrido. Além disso, o método proposto deverá ser comparado com abordagens de CNN.

\section{Referências}

Al-Akaidi, M. (2004). Fractal speech processing. Cambridge university press.

Araujo, A. F. et al. (2012). Análise e caracterização de lesões de pele para auxílio ao diagnóstico médico. Avanços em Visão Computacional.

Bhati, P. e Singhal, M. (2015). Early stage detection and classification of melanoma. In Communication, Control and Intelligent Systems (CCIS), 2015, pages 181-185. IEEE.

Breiman, L. (2001). Random forests. Machine learning, 45(1):5-32.

Chang, W.-Y. et al. (2013). Computer-aided diagnosis of skin lesions using conventional digital photography: a reliability and feasibility study. PloS one, 8(11):e76212.

Chu, A., Sehgal, C. M., e Greenleaf, J. F. (1990). Use of gray value distribution of run lengths for texture analysis. Pattern Recognition Letters, 11(6):415-419.

Codella, N., Cai, J., et al. (2015). Deep learning, sparse coding, and svm for melanoma recognition in dermoscopy images. In International Workshop on Machine Learning in Medical Imaging, pages 118-126. Springer.

Cortes, C. e Vapnik, V. (1995). Support-vector networks. Machine learning, 20(3):273297.

Dalal, N. e Triggs, B. (2005). Histograms of oriented gradients for human detection. In Computer Vision and Pattern Recognition, 2005. CVPR 2005. IEEE Computer Society Conference on, volume 1, pages 886-893. IEEE.

Dasarathyand, B. R. e Holder, E. B. (1991). Image characterizations based on joint graylevel run-length distributions. Pattern Recognition Letters, 12(8):497-502.

Diepgen, T. L. e Yihune, G. (2012). Dermatology information system - dermis. http: //dermis.net/. Acessado em: 2017.

Galloway, M. M. (1975). Texture analysis using gray level run lengths. Computer graphics and image processing, 4(2):172-179. 
Haralick, R. M., Shanmugam, K., et al. (1973). Textural features for image classification. IEEE Transactions on systems, man, and cybernetics, 3(6):610-621.

Haykin, S. S. (2009). Neural networks and learning machines, volume 3. Pearson Upper Saddle River, NJ, USA:.

INCA (2016). Tipo - pele melanoma. http://www.inca.gov.br/wps/wcm/ connect/tiposdecancer/site/home/pele_melanoma. Acessado em: 2017.

Kumar, A. et al. (2016). An ensemble of fine-tuned convolutional neural networks for medical image classification. IEEE Journal of Biomedical and Health Informatics.

Lacy, K. e Alwan, W. (2013). Skin cancer. Medicine, 41(7):402-405.

Landis, J. R. e Koch, G. G. (1977). The measurement of observer agreement for categorical data. biometrics, pages 159-174.

Mendonça, T. et al. (2013). Ph 2-a dermoscopic image database for research and benchmarking. In Engineering in Medicine and Biology Society (EMBC), 2013 35th Annual International Conference of the IEEE, pages 5437-5440. IEEE.

Moura, N. H. et al. (2015). Classificação de lesões de pele utilizando atributos de cor e textura. Workshop de Informática Médica - WIM.

Ojala, T., Pietikäinen, M., e Harwood, D. (1996). A comparative study of texture measures with classification based on featured distributions. Pattern recognition, 29(1):51-59.

Oliveira, R. B. et al. (2016). A computational approach for detecting pigmented skin lesions in macroscopic images. Expert Systems with Applications, 61:53-63.

Rosenfield, G. H. e Fitzpatrick-Lins, K. (1986). A coefficient of agreement as a measure of thematic classification accuracy. Photogrammetric engineering and remote sensing, 52(2):223-227.

Sánchez-Monedero, J. et al. (2016). Classification of melanoma presence and thickness based on computational image analysis. In International Conference on Hybrid Artificial Intelligence Systems, pages 427-438. Springer.

Shin, H.-C., Roth, H. R., et al. (2016). Deep convolutional neural networks for computeraided detection: Cnn architectures, dataset characteristics and transfer learning. IEEE transactions on medical imaging, 35(5):1285-1298.

Silva Júnior, E. R. (2007). Investigação de técnicas de extração e seleção de características e classificadores aplicados ao problema de classificação de dígitos manuscritos de imagens de documentos históricos. $51 \mathrm{f}$. Monografia (Bacharelado em Engenharia da Computação) - Escola Politécnica de Pernambuco, UFPE, Pernambuco.

Sobel, I. (1970). Camera models and machine perception. Technical report, DTIC Document.

Society, A. C. (2015). Cancer facts \& figures 2015. http://cancer. org/acs/groups/content/ deditorial/documents/document / acspc-0 44552 .pdf. Acessado em: 2017.

Tamura, H., Mori, S., e Yamawaki, T. (1978). Textural features corresponding to visual perception. IEEE Transactions on Systems, Man, and Cybernetics, 8(6):460-473. 